\title{
O ptimisation du réseau algérien de transport de brut et de condensat
}

\author{
A. Smati ${ }^{1}$, N. Zeraibi ${ }^{1}$ et M.C. Touabti \\ 1 Université de Boumerdes, Institut des hydrocarbures et de la chimie, Département énergétique, Boumerdes 35000 - Algérie \\ 2 Université de Sétif, Laboratoire de génie du gaz, Sétif 19000 - Algérie \\ e-mail : a.smati@yahoo.com
}

Résumé - Le diagnostic et l'extension optimale du réseau algérien de transport de brut et de condensat, en liaison avec l'intensification des rythmes de production, nécessitent la mise au point de procédures systémiques de modélisation. Dans ce contexte, le pompage par batch est représenté par des bi-flots. L'arsenal de la théorie des graphes et le problème du flot maximal, associé à un modèle de fiabilité, sont ensuite mis à contribution. Le modèle global peut alors être utilisé comme stand d'expérimentation où l'impact de chaque décision ou événement est dégagé par simulation. Cependant, en raison de la nonlinéarité des coûts, l'extension optimale du réseau est soumise à la résolution d'un modèle de programmation non-linéaire.

Mots-clés : optimisation, multiproduit, flot maximal, bi-flots, fiabilité, programmation non-linéaire.

\begin{abstract}
Optimization of the Algerian Crude and Condensate Network - The diagnosis and the optimal extension of the Algerian crude and condensate network, as a result of the escalation of production rhythms, necessitate the development of systemic procedures of modeling. In this context, the batching is represented by a two-commodity network flow. The theory of graphs and the maximal flow problem, associated with a model of reliability, are then put to contribution. The global model can then be used as an experimentation stand where the impact of any decision or event is released by simulation. However, because of the nonlinearity of costs, the optimal extension of the network is submitted to the resolution of a nonlinear programming model.
\end{abstract}

Keywords: optimization, batching, maximal flow, two-commodity network flows, reliability, nonlinear programming. 


\section{IN TRO DUCTION}

Le réseau algérien de transport d'hydrocarbures liquides relie par canalisations les champs sahariens de production de brut et de condensat aux sites d'exportation et de raffinage implantés sur le littoral (fig. 1). Les prévisions de production, en relation à l'ouverture du secteur aux compagnies internationales et à la découverte de nouveaux gisements de brut et de gaz humide, se démarquent par une augmentation substantielle de la production globale de brut et de condensat. Ces perspectives sous-tendent impérativement l'élaboration, à court et moyen termes, de nouveaux schémas de transport incluant :

- des décisions synchroniques de réaffectation des flux d'expédition par les différentes lignes et la possibilité d'une généralisation du pompage par batch sur plusieurs lignes ;

- des décisions diachroniques caractérisées par des actions de réhabilitation des équipements existants, la construction de capacités de stockage supplémentaires et la réalisation de nouvelles lignes.

Dans cette optique, il y a lieu de développer des outils méthodologiques adaptés permettant de répondre aux questions essentielles suivantes :

- le réseau, dans sa configuration actuelle, peut-il assurer l'évacuation des quantités prévues de brut et de condensat?

- quels sont les goulots d'étranglement et où se situent-ils ?

- quelles sont les décisions techniques à prendre sur l'ensemble du réseau pour assurer les objectifs à moindre coût ?
- sur quelles lignes le batching doit-il être effectué ?

- quelles sont les réserves du réseau dans sa configuration finale, et où se situent-elles ?

Dans ce cadre, il y a donc lieu de développer une approche en trois étapes :

- diagnostic du réseau dans sa configuration actuelle ;

- analyse de la fiabilité du réseau et de l'incidence des actions de réhabilitation ;

- extension optimale du réseau.

\section{MODÉLISATION DU RÉSEAU DE TRAN SPORT DE BRUT ET DE CONDENSAT}

Pour apporter des éléments de réponse aux questions précédentes, il est nécessaire de procéder à la modélisation du réseau dans sa globalité. Le réseau algérien de transport de brut et de condensat est un système complexe. Jusqu'à présent, on a le plus souvent tenté d'améliorer les performances des systèmes complexes en optimisant les performances partielles, fonction par fonction. Cette approche, dite marginale [1], ignorant les interactions, ne peut satisfaire le décideur pour au moins deux raisons :

- la compatibilité des décisions locales et la cohérence du système global ne sont pas assurées ;

- dans le cadre d'une optimisation intégrée, un ensemble de solutions optimales locales ne constitue pas forcément un optimum global.

Il y a donc lieu de privilégier une perception systémique du réseau [2-4]. Pour cela, il est nécessaire de développer une

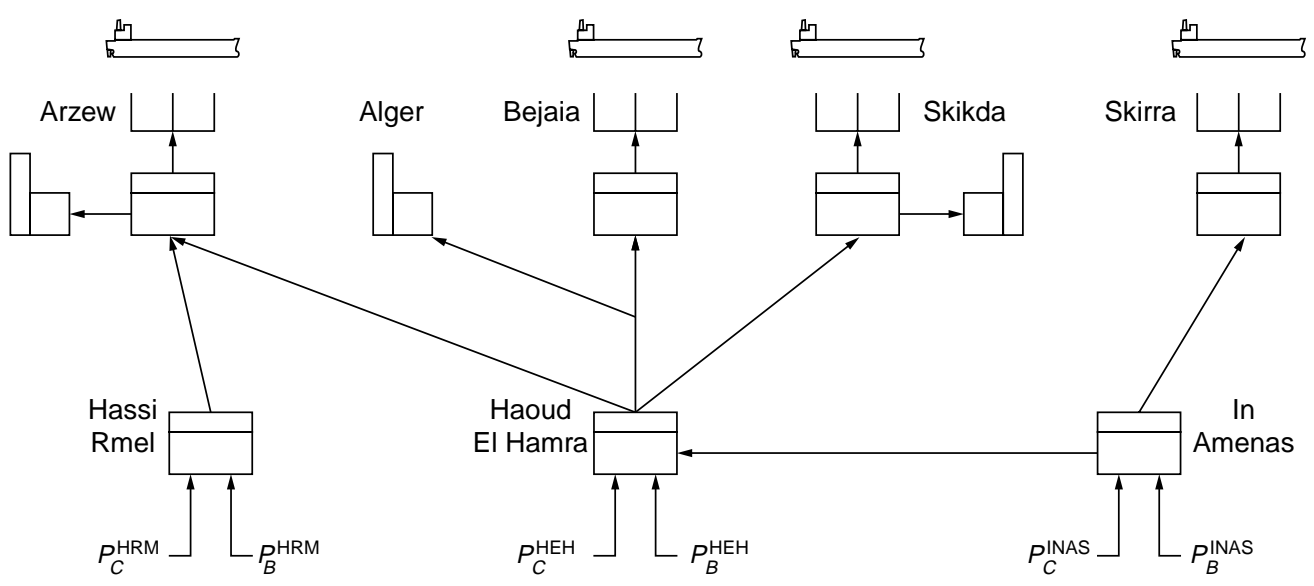

Figure 1

Schéma simplifié du réseau algérien de transport de brut et de condensat.

Simplified diagram of the Algerian transportation system of crude and condensate. 
représentation mathématique de l'ensemble du système, intégrant les événements et les décisions qui façonnent la performance et sous-tendent les évaluations et le diagnostic. La modélisation analytique du réseau, dans son ensemble et dans ses moindres détails, est d'une très grande complexité. Ceci en raison principalement [5-7] :

- du très grand nombre de variables à mettre en jeu ;

- des différentes natures physiques des processus technologiques entrant dans la composition de la chaîne de transport (production, stockage, oléoduc, port, flotte);

- de la difficulté de coordonner les interactions dynamiques entre les représentations mathématiques des différents processus.

Dans ce contexte, la modélisation par la théorie des graphes et la mise à contribution du problème du flot maximal [8-12] offrent une alternative simple et efficace, pour peu que les flux puissent être exprimés par un même ensemble d'unités.

Un graphe $G=[X, U]$ est déterminé par :

- un ensemble $X$ dont les éléments sont appelés sommets ou nœuds, le nombre de nœuds définissant l'ordre du graphe ;

- un ensemble $U$ dont les éléments sont des couples ordonnés de sommets, appelés arcs.

Cependant, l'option batching nécessite l'introduction de la notion complexe de multiflots [11, 13, 14]. Pour contourner cet épineux problème, nous userons d'un artifice, s'apparentant au concept d'ensemble déconnectant [14], pour ramener la modélisation du pompage par batch à une représentation classique à flot simple, ce qui nous permettra de mettre à profit le problème du flot maximal pour la simulation de différentes variantes et la synthèse d'un schéma de transport optimal à moyen terme.

Le batching est une technique de pompage consistant à expédier deux ou plusieurs produits pétroliers différents, séparément et dans un ordre déterminé, par une même conduite. Le batching est le plus souvent utilisé lorsque les quantités de produits transportées ne peuvent justifier la construction de canalisations séparées pour chaque produit. Il permet, entre autres, d'augmenter le taux d'utilisation du pipeline, de diminuer les frais d'investissement et surtout d'améliorer la flexibilité du réseau. La flexibilité est devenue un trait caractéristique important de l'organisation des systèmes industriels confrontés à un environnement changeant. Pour être flexible, un système doit développer des formes plus complexes d'organisation, qui incorporent une grande variété de solutions et une capacité d'adaptation aux différentes situations qui peuvent se présenter (panne prolongée de certains équipements, fluctuation de la demande, etc.).

Une chaîne de transport de pétrole brut se compose (fig. 2a) :

- d'une ou de plusieurs sources ;

- d'un parc de stockage « départ »;

- de l'oléoduc proprement dit ;
- d'un terminal de stockage « arrivée »;

- d'un port pétrolier composé de plusieurs postes de chargement ;

- d'une flotte de tankers pour l'exportation ;

- souvent, d'une livraison vers une raffinerie.

La représentation sous forme de graphe d'une telle chaîne, dans le cas où les clients sont assimilés à un puits unique, est donnée par la figure $2 \mathrm{~b}$. Dans ce schéma :

- l'arc $(s, 1)$ représente la source de production ;

- l'arc $(1,2)$, la capacité de stockage du terminal « départ »;

- l'arc (2, 3), le tronçon de conduite en amont du point de piquage ;

- l'arc $(3,4)$, le tronçon de conduite en aval du point de piquage ;

- l'arc $(4,5)$, la capacité de stockage du terminal «arrivée »;

- les arcs $(5,6),(5,7)$ et $(5,8)$, les postes de chargement du port pétrolier.

À chaque arc sont affectées les capacités maximale et minimale du flot qui le traverse. Pour ramener les flux à un même ensemble d'unités, les flux des arcs représentant les stockages sont définis, au stade de l'avant-projet, par l'intermédiaire d'une heuristique, largement utilisée en pratique, admettant que les capacités de stockage correspondent à l'équivalent de sept jours de pompage avec le débit nominal. L'arc $(3, t)$, représentant l'alimentation ininterrompue et prioritaire de la raffinerie, se verra affecter une seule valeur, correspondant à la demande de la raffinerie. Les arcs correspondant aux postes de chargement d'un port peuvent être représentés par un arc équivalent unique. Cependant, en raison de leur mode de fonctionnement parallèle et indépendant, la représentation adoptée permet l'analyse des risques de saturation par poste et surtout celle de l'impact d'une mise hors service d'un poste de chargement sur l'ensemble du réseau.

Une chaîne de transport de pétrole brut, moyennant quelques adaptations mineures des ouvrages de stockage, peut être, le cas échéant, utilisée pour le transport par batch. En conséquence, on admettra que toute chaîne pourra tout aussi bien servir au batching qu'au pompage d'un produit unique. La modélisation par la théorie des graphes du pompage par batch du brut et du condensat induit l'introduction de la notion de bi-flots [13, 14]. La chaîne est ramenée à une représentation à flot simple par l'introduction d'un ensemble d'artifices :

- à chaque flot on associe comme support un sous-graphe partiel ;

- à chaque flot est associée une source spécifique ;

- un arc artificiel est introduit pour borner la capacité maximale de la ligne.

Cela se traduit par la représentation sagittale de la figure $2 \mathrm{c}$, où :

- l'arc $(s, 1)$ représente la source de production de condensat;

- l' $\operatorname{arc}(s, 2)$, la source de production de brut ; 

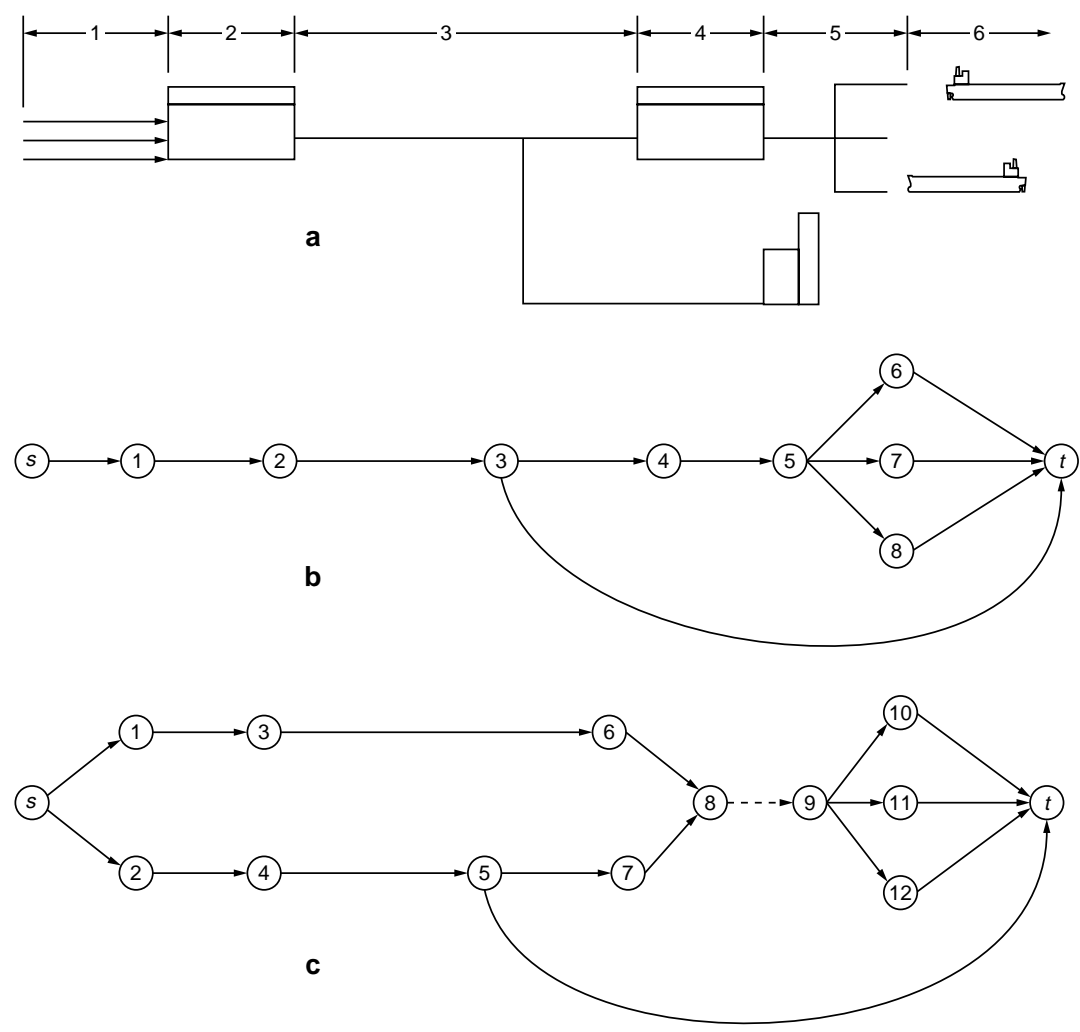

Figure 2

Représentation par les graphes d'une chaîne de transport de deux produits par batch.

Representation by graphs of a transportation chain of two products by batch.

- les arcs $(1,3)$ et $(2,4)$, les capacités de stockage en condensat et en brut du terminal « départ »;

- les arcs $(6,8)$ et $(7,8)$, les capacités de stockage en condensat et en brut du terminal «arrivée »;

- l'arc $(8,9)$, un arc artificiel déconnectant.

La modélisation d'un réseau complexe de transport de brut et de condensat disposant de plusieurs chaînes se basera sur les graphes élémentaires définis plus haut. La modélisation du réseau algérien dans son schéma de fonctionnement actuel (batching sur une seule ligne) est donnée en figure 3. Dans un contexte d'options stratégiques, la décision n'est pas subordonnée à un calcul classique de rentabilité. Le problème consiste à trouver un flot dans le graphe qui satisfasse au mieux les besoins des clients compte tenu des disponibilités. Pour cela, le graphe est transformé en réseau de transport, dans le sens de la théorie des graphes, par la création de :

- une entrée fictive $s$ que l'on appellera supersource;

- une sortie fictive $t$ que l'on assimilera à un superpuits ;

- un arc de retour fictif $(t, s)$ auquel, par convention, on attribuera le numéro 0 .

Le problème consiste alors à déterminer un flot $\varphi=\left[\varphi_{0}\right.$, $\left.\varphi_{1}, \ldots, \varphi_{M}\right]^{T}$ dans le graphe vérifiant les contraintes de capacités $C_{u}^{-} \leq \varphi_{u} \leq C_{u}^{+}(\forall u \in U)$ et tel que la composante $\varphi_{0}$ sur l'arc de retour soit maximale :

$$
\begin{gathered}
\max \varphi_{0}(t, s) \\
C_{u}^{-} \leq \varphi_{u} \leq C_{u}^{+} \\
\sum_{u \in d_{u}^{+}} \varphi_{u}=\sum_{u \in d_{u}^{-}} \varphi_{u}
\end{gathered}
$$

avec $d_{u}^{+}$, demi-degré extérieur du nœud $u$ et $d_{u}^{-}$, demi-degré intérieur du nœud $u$.

Le problème du flot maximal dans un graphe a donné naissance à de nombreux algorithmes qui, dans leur logique, utilisent la structure particulière des matrices de ce type de problème. Le plus connu est l'algorithme de Ford-Fulkerson [9]. Cependant, les problèmes de flots étant des programmes linéaires, la résolution peut être également obtenue par l'intermédiaire de l'algorithme du simplexe ou l'une de ses variantes $[12,15,16]$. La solution du problème ainsi posé permettra de répondre, par simple comparaison entre $\varphi_{0}$ et la demande $d$ (ou la production), à la première question relative aux capacités du réseau, concernant l'expédition des quantités prévues. 


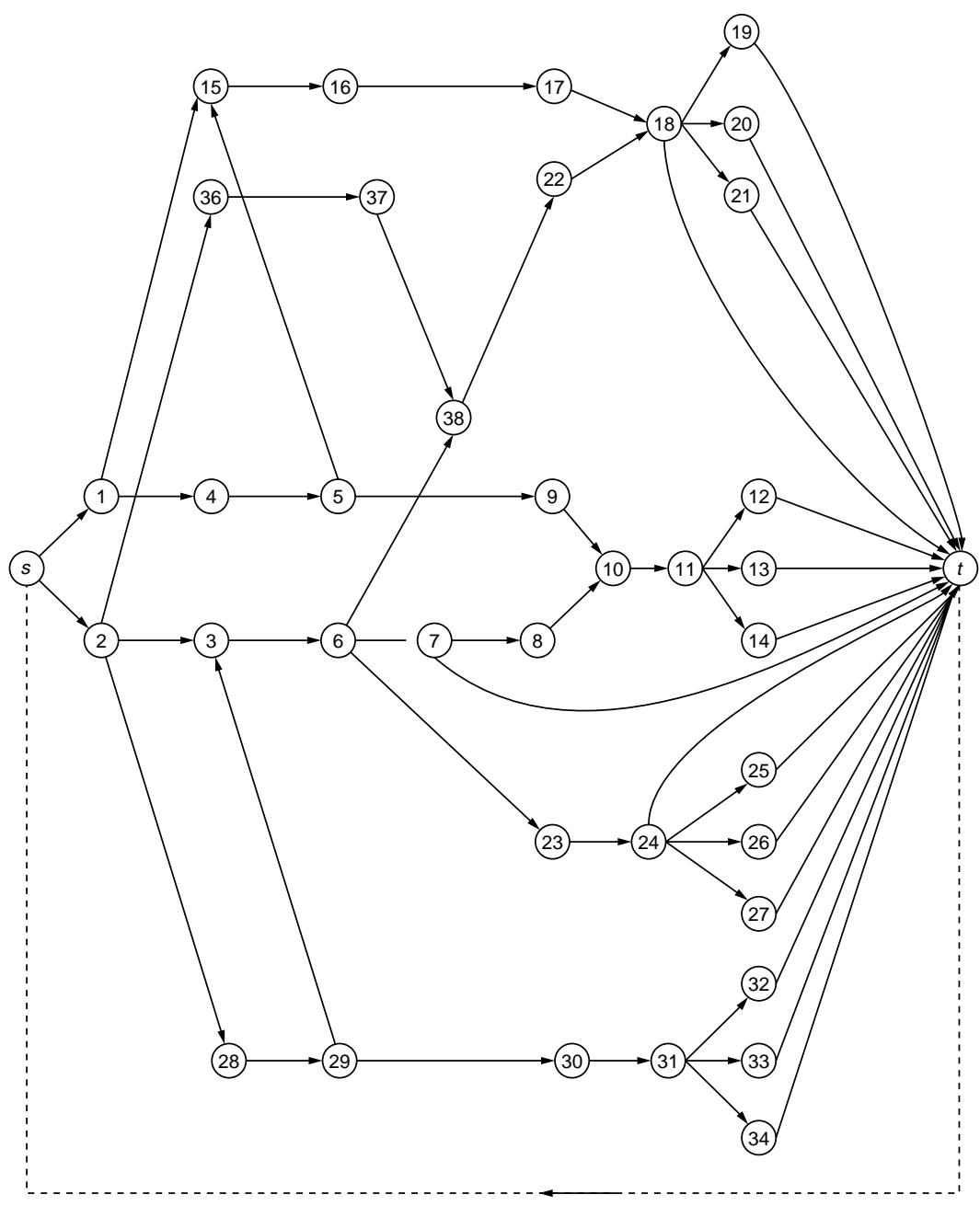

Figure 3

Graphe du réseau algérien de transport de brut et de condensat dans son fonctionnement actuel.

Graph of the Algerian transportation network of crude and condensate in its current functioning.

\section{MODÈLE DE FIABILITÉ DU RÉSEAU}

La mission essentielle d'un réseau de transport est d'établir un flot, entre le nœud source $s$ et le nœud puits $t$, supérieur ou égal à la demande. Dans ce contexte, les éléments principaux du réseau sont manifestement les arcs représentant les oléoducs. Cependant, les capacités maximales des lignes $C_{u}^{+}$ fluctuent de manière aléatoire et évoluent en fonction du temps dans le sens d'une diminution des performances, en raison principalement :

- des pannes intempestives et de l'obsolescence des équipements de pompage ;

- de l'encrassement, de la corrosion et de l'évolution de la rugosité interne de la conduite.

La conséquence immédiate en est un risque de déséquilibre dans les liaisons entre les différents éléments et le passage du système global à un niveau inférieur d'homéostasie. De plus, le vieillissement inégal des lignes, en liaison avec la date de leur mise en service, fait que les chutes de performance sont différentes d'un ouvrage à l'autre. En conséquence, la condition $\varphi_{0} \geq d$ est un événement aléatoire dont la réalisation dépend de la fiabilité des éléments principaux composant le réseau. En assimilant l'oléoduc à un modèle «boîte noire» soumis au vecteur des perturbations $\dot{Z}(t)$, la fiabilité de la ligne $i$ s'exprime par l'intermédiaire de la relation suivante (fig. 4) :

$$
R_{i}=\frac{\int_{0}^{T} q_{i}(t) \mathrm{d} t}{q_{0 i} \int_{0}^{T} \mathrm{~d} t}
$$

$\dot{Z}(t)$ représente les perturbations provoquées par les pannes intempestives et $\dot{X}(t)$, vecteur des paramètres de commande, 


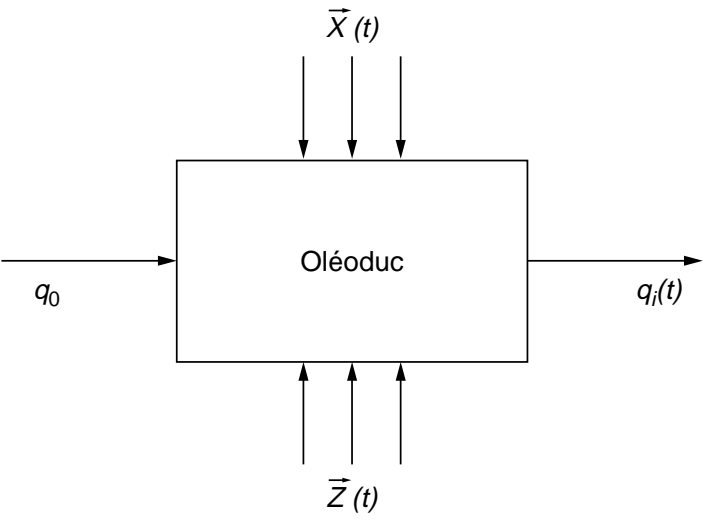

Figure 4

Représentation «boîte noire » du fonctionnement d'un oléoduc.

"Black box" representation of a pipeline.

les réactions de l'opérateur face aux perturbations. La fiabilité dans ce contexte signifie la mesure de l'efficacité et de la performance du système.

Sur la base de données antérieures d'exploitation, la fiabilité opérationnelle de la ligne $i$ peut alors être estimée comme suit :

$$
R_{i}^{\mathrm{op}}=\frac{C_{r i}}{C_{0 i}}
$$

avec $C_{r i}$, quantités de produit réellement transportées par la ligne $i$ et $C_{0 i}$, capacité nominale (design) de la ligne $i$ :

$$
\begin{gathered}
C_{0 i}=q_{0 i} T_{i} \\
C_{r i}=\sum_{S_{i}} q_{i j} \tau_{i j}
\end{gathered}
$$

avec :

$q_{0 i} \quad$ débit nominal de la ligne $i$

$T_{i} \quad$ durée d'expérimentation

$S_{i} \quad$ dimension de la gamme des débits de pompage de la ligne $i$

$q_{i j} \quad j$-ième débit de la gamme $S_{i}$

$\tau_{i j} \quad$ durée de pompage totale avec le débit $q_{i j}$

et :

$$
T_{i}=\sum_{S_{i}} \tau_{i j}
$$

D'où :

$$
R_{i}^{\mathrm{op}}=\frac{1}{q_{0 i}} \sum_{S_{i}} \frac{q_{i j} \tau_{i j}}{T_{i}}=\frac{1}{q_{0 i}} M\left\{q_{i}\right\}
$$

Pour un réseau complet, assimilé à une boîte noire équivalente, la fiabilité opérationnelle peut alors être exprimée par la relation suivante:

$$
R_{\mathrm{res}}^{\mathrm{op}}=\frac{\varphi_{0}^{\mathrm{op}}}{\varphi_{0}^{\text {nom }}}
$$

avec $\varphi_{0}{ }^{\text {op }}$, flot maximal du réseau en tenant compte des capacités réelles $C_{r i}^{+}, \varphi_{0}{ }^{\text {nom }}$, flot maximal du réseau en affectant aux lignes leurs capacités nominales $\mathrm{C}_{0 i}^{+}$et :

$$
C_{r i}^{+}=R_{i} C_{0 i}^{+}
$$

Cependant, la relation simple (5), très utilisée en pratique, ne distingue pas les baisses de régime dues aux pannes de celles, volontaires, dictées par les conditions d'exploitation. De plus, elle ne permet pas d'établir de liaisons explicites entre les actions susceptibles d'améliorer la fiabilité de l'ouvrage (rénovation de certaines pompes ou redondance par exemple) et la capacité de transit. Il est clair que dans notre cas, il faudrait rechercher l'expression de la fiabilité intrinsèque ou prévisionnelle de chaque ouvrage. La fiabilité prévisionnelle est définie comme « la fiabilité calculée sur la base d'un modèle mathématique conçu à partir des données de projet et de la fiabilité estimée des composants » [17].

\subsection{Fiabilité d'un oléoduc}

Un oléoduc est constitué d'un certain nombre de stations de pompage (SP), distantes d'une centaine de kilomètres les unes des autres (fig. 5). La fonction principale des SP est de fournir l'énergie de pression nécessaire à l'écoulement du produit dans la conduite. Chaque SP est constituée de plusieurs pompes, assemblées en série ou en parallèle, et d'une ou deux pompes de réserve. En fonctionnement normal, la pompe de réserve est à l'arrêt. Elle est activée dès qu'une panne se produit sur un élément (redondance passive). En cas de défaillance d'une station intermédiaire (ou d'une pompe), celle-ci peut être bipassée en attendant sa réparation. Mais toute panne entraîne une diminution du débit de pompage. L'ampleur de cette chute de performance est directement liée à la nature de la défaillance et à son emplacement. La nature intempestive des défaillances entraîne une variation aléatoire du débit d'exploitation. Ces fluctuations peuvent alors être probabilisées suivant une loi de distribution statistique, ou assimilées à un processus aléatoire à espace de phase discret. Dans les deux cas, la perte de performance due aux pannes de l'équipement peut être quantifiée comme suit :

$$
R_{1}=\frac{1}{q_{0}} \sum_{s} P_{i} q_{i}
$$

où $P_{i}$ est la probabilité de la panne $i$ et $q_{i}$ le débit maximal de la ligne en présence de la panne $i\left(q_{i}<q_{0}\right)$. 


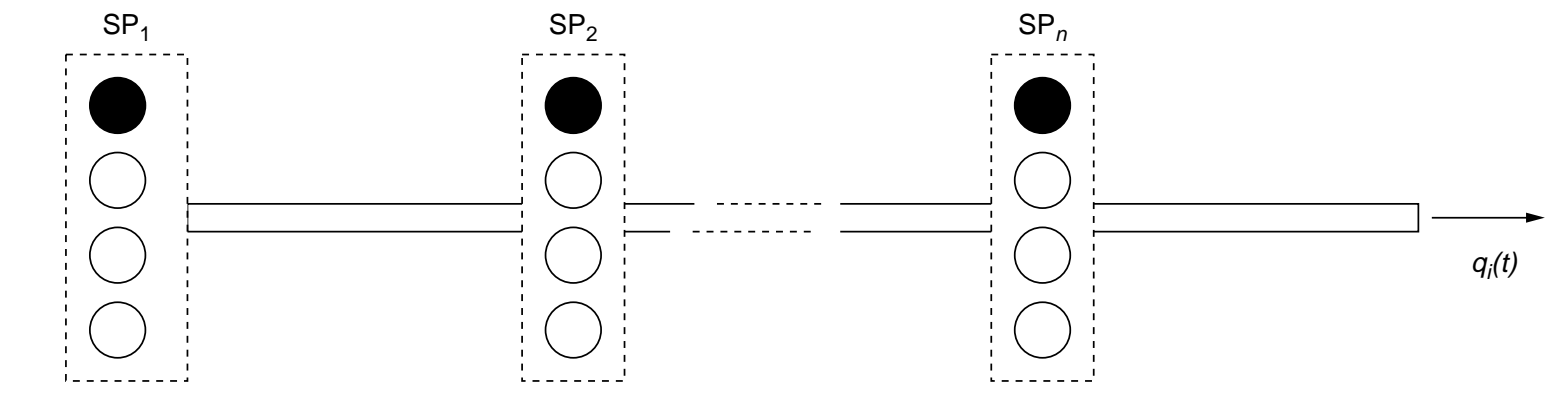

Figure 5

Schéma général d'un oléoduc composé de $n$ stations de pompage.

General diagram of a pipeline composed of n pumping stations.

Pour être complète, la caractéristique de fiabilité introduite doit tenir compte également du risque d'arrêt complet de la ligne (débit nul). L'arrêt complet de la ligne peut se produire dans deux cas - défaillance complète de la station de départ ou incident sur la canalisation (fuite par exemple) :

$$
R=\frac{1-P}{q_{0}} \sum_{s} P_{i} q_{i}
$$

où $P$ représente la probabilité d'arrêt complet de la ligne.

Le coefficient de fiabilité caractérise la faculté (la probabilité) du système à accomplir correctement ses tâches lorsqu'il est sollicité. Le débit nominal $q_{0}$ est une valeur constante connue, caractérisant les performances de l'oléoduc lors d'un fonctionnement sans défaillances. Les débits $q_{i}$, correspondant à chaque point de phase (à chaque type de panne), peuvent être déterminés en utilisant les relations hydrauliques décrivant l'écoulement d'un fluide incompressible dans une conduite et les caractéristiques des pompes en liaison avec le type d'assemblage. Le calcul des probabilités de phase $P_{i}$ et de la probabilité de défaillance complète de la ligne $P$ se base sur le traitement statistique de l'historique des pannes et de leur durée. Ce calcul nécessite la détermination des indices de fiabilité des principaux éléments du système.

\subsection{Estimation des indices de fiabilité}

Les principaux indices de fiabilité des éléments constitutifs d'un oléoduc sont :

- le taux de défaillance des pompes $r_{P}$, des SP $r_{S P}$ et de la conduite $r_{l}$;

- le taux de réparation de chaque élément $h_{P}, h_{S P}$ et $h_{l}$.

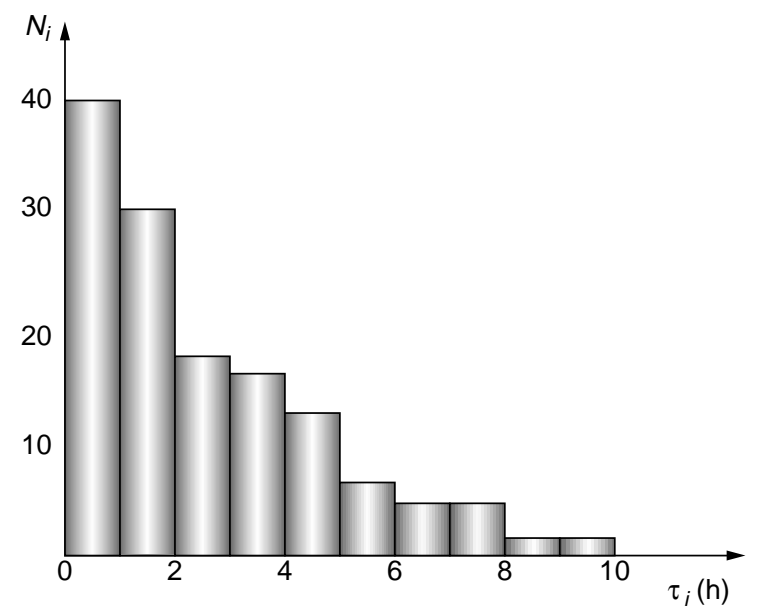

Figure 6

Histogramme des pannes en fonction de la durée de réparation des pompes de l'oléoduc HEH-Bejaia.

Histogram of failures as a function of the duration of a pump repair in the HEH-Bejaia pipeline.

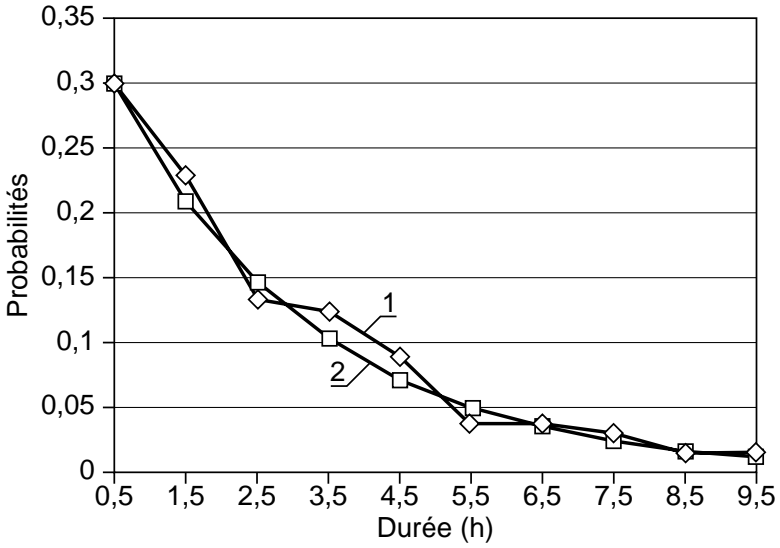

Figure 7

Distributions réelle (1) et théorique (2) des probabilités de panne en fonction de la durée de réparation.

Real (1) and theoretical (2) distributions of probabilities of failure in function of the repair duration. 
TABLEAU 1

Indices de fiabilité de l'oléoduc HEH-Bejaia

Reliability indices of the HEH-Bejaia pipeline

\begin{tabular}{l|c|c|c}
\hline Éléments & $\begin{array}{c}\text { Taux de pannes } r \\
\left(\mathrm{~h}^{-1}\right)\end{array}$ & $\begin{array}{c}\text { Durée moyenne } \\
\text { de réparation }\end{array}$ \\
\hline Pompes & 0,00178 & $2 \mathrm{~h} 48 \mathrm{~min}$ \\
Stations de pompage & 0,00165 & $0 \mathrm{~h} 50 \mathrm{~min}$ & 0,356 \\
Conduites & 0,00068 & $51 \mathrm{~h} 00 \mathrm{~min}$ & 1,195 \\
\hline
\end{tabular}

Si l'on suppose l'apparition d'une panne comme un événement indépendant, la superposition d'un grand nombre de flots d'événements conduit le plus souvent à des flots poissoniens pour lesquels la durée entre deux événements successifs obéit à une loi exponentielle (fig. 6 et 7). La loi exponentielle jouit de la remarquable propriété d'absence de mémoire et c'est la seule loi à taux de défaillance constant :

$$
r=\frac{N_{d}}{T_{f}}
$$

où $N_{d}$ est le nombre total de pannes se produisant durant la période de fonctionnement effectif $T_{f}$.

Le même raisonnement peut également être appliqué à la durée moyenne de réparation :

$$
\tau_{r}=\frac{1}{N_{d}} \sum_{i=1}^{N_{d}} \tau_{r i}
$$

avec :

$$
h=\frac{1}{\tau_{r}}
$$

et $\tau_{r i}$, durée de réparation de la panne $i$ et $h$, taux moyen de réparation.

L'hypothèse d'exponentialité simplifie les calculs. Les résultats obtenus revêtent une forme commode et facilement interprétable. Ainsi, le taux de panne peut être interprété comme la probabilité de voir l'élément tomber en panne lorsqu'il est sollicité. Le taux de réparation caractérise la capacité de prévention, de détection et d'élimination de la défaillance. L'estimation des indices de fiabilité pour l'oléoduc HEH-Bejaia est donnée dans le tableau 1.

\subsection{Calcul des probabilités de phase}

Si toutes les stations de pompage d'un oléoduc sont identiques, en utilisant la structure dite de « $k$ parmi $n$ éléments indépendants », la probabilité d'avoir simultanément $k$ SP hors service se calcule par la relation :

$$
Q_{k}=\frac{C_{n-1}^{k} r_{S P}^{k} h_{S P}^{n-k-1}}{\left(r_{S P}+h_{S P}\right)^{n-1}}
$$

Pour une SP composée de $m$ pompes principales et $l$ pompes de réserve, sur la base de la théorie de naissance et de mort, les différentes situations de panne, ou plus exactement leurs probabilités, sont calculées à l'aide du modèle suivant $[7,18]$ :

$$
\begin{array}{ll}
P_{k}=\frac{\left(r_{p} / h_{p}\right)}{k !} P_{0} & \text { si } k \leq l \\
P_{k}=m^{l} \frac{\left(r_{p} / h_{p}\right)}{k !} \prod_{j=0}^{k-l-1}(m-j) P_{0} & \text { si } k>l
\end{array}
$$

avec :

$$
\frac{1}{P_{0}}=\sum_{k=0}^{l} m^{k} \frac{\left(r_{p} / h_{p}\right)^{k}}{k !}+m^{l} \sum_{k=l+1}^{m+l} \frac{\left(r_{p} / h_{p}\right)^{k}}{k !} \prod_{j=0}^{k-l-1}(m-j)
$$

Le tableau 2 donne les résultats du calcul des probabilités élémentaires statistiquement significatives pour l'oléoduc HEH-Bejaia.

La SP sera considérée en situation de non-défaillance tant que le nombre de pompes en panne sera inférieur au nombre de pompes de réserve. De plus, la probabilité d'un arrêt complet de la ligne, suite à une mise hors service de la station de départ ou à un incident sur la canalisation, se calculera comme la somme des probabilités de ces deux événements. Pour des oléoducs de faible ou moyenne longueur, tels que les oléoducs algériens, il s'avère peu probable d'avoir une panne simultanée de plus de deux éléments. Ceci permet de ramener l'espace de phase à des dimensions parfaitement gérables. On ne retiendra donc que les événements suivants pour tous les $i=1 \div n$ et tous les $j=1 \div n$ :

- $E_{0}$, disponibilité de toutes les SP et des pompes principales dans toutes les SP ;

- $E 1_{i}$, disponibilité de toutes les SP, une pompe dans la SP $i$ tombe en panne ;

- $E 2 j$, une SP parmi les $j$ est hors service, toutes les pompes principales sont disponibles ;

- $E 3_{j i}$, la SP $j$ est hors service, ainsi qu'une pompe principale dans la SP $i$.

La probabilité de chaque événement est calculée sur la base des probabilités élémentaires. La somme des probabilités de ces événements doit être très proche de 
TABLEAU 2

Probabilités élémentaires des événements statistiquement significatifs de l'oléoduc HEH-Bejaia

Elementary probabilities of the statistically significant events of the HEH-Bejaia pipeline

\begin{tabular}{l|c|c}
\hline Phases & Désignation & Probabilité \\
\hline Toutes les SP en bon état de fonctionnement & $Q_{0}$ & 0,9980 \\
Une SP tombe en panne & $Q_{1}$ & 0,0140 \\
Toutes les pompes principales en état de marche & $P_{0}$ & 0,9802 \\
Une pompe dans une des SP tombe en panne & $P_{1}$ & 0,0049 \\
\hline
\end{tabular}

TABLEAU 3

Fiabilité et capacité maximale de transit pour différents scénarios

Reliability and maximal transit capacity for different scenarios

\begin{tabular}{l|c|c}
\hline Scénarios de simulation & $R_{i}$ & $C_{i}^{+}$(MTA) \\
\hline Fiabilité actuelle & 0,9566 & 16,262 \\
En période de vieillissement & 0,9200 & 15,64 \\
Réduction de la durée de réparation de 48 min & 0,9668 & 16,435 \\
Rénovation de toutes les pompes & 0,9744 & 16,565 \\
Rénovation + réduction de la durée de réparation & 0,9800 & 16,660 \\
\hline
\end{tabular}

l'unité. Si cette condition n'est pas vérifiée, on doit inclure des événements à probabilité de réalisation plus faible (panne simultanée de trois éléments). Ce cas de figure peut se rencontrer dans des oléoducs composés d'un nombre élevé de SP, ou de SP en phase de vieillissement. Ce modèle permet de quantifier l'impact de toute décision tendant à augmenter la fiabilité sur la valeur maximale du flux de la ligne. Les résultats de certaines variantes d'action pour l'oléoduc HEH-Bejaia sont donnés dans le tableau 3.

\section{SIMULATION DES DÉCISIONS}

Le modèle ainsi obtenu, associant la modélisation par le flot maximal au modèle de fiabilité, pourrait alors être utilisé comme stand d'expérimentation et, de ce fait, permettre :

- la simulation de l'impact de toute décision de réaffectation des flux, de rénovation partielle ou d'extension du réseau ;

- la détermination des décisions d'extension nécessaires pour assurer l'expédition des quantités désirées.

Cependant, pour le second point, la résolution par simulation heuristique peut nécessiter l'examen d'un nombre prohibitif de scénarios. Cet inconvénient est commun à tous les problèmes usant de la simulation comme outil d'optimisation. En conséquence, il est nécessaire de développer un plan d'expériences permettant de réduire le nombre de variantes à examiner. Ce procédé est connu en systémique sous l'appellation de principe de Rodin. À une question relative à sa technique de travail, le célèbre sculpteur aurait ironisé : «J'enlève tout ce qui est superflu. » Dans notre cas, il s'agira donc de simuler principalement les scénarios consistant à élargir les goulots d'étranglement. Pour la localisation des goulots d'étranglement éventuels, on utilisera la règle de coloration suivante :

- l'arc de retour et les arcs fictifs de l'ensemble déconnectant en noir ;

- un arc $u \neq 0$ tel que $\varphi_{u}=0$ en noir ;

- les arcs à valeur unique (raffineries) en noir ;

- un arc $u \neq 0$ tel que $0<\varphi_{u}<c_{u}$ en rouge ;

- un arc $u \neq 0$ tel que $\varphi_{u}=c_{u}$ en vert.

La résolution du problème par le critère du flot maximal, associée à cette coloration, fera apparaître les goulots d'étranglement en vert et les réserves potentielles en rouge. Pour définir les capacités supplémentaires à installer, les arcs verts seront affectés d'un nombre $M$ très grand. La valeur d'écart $\varphi_{u}^{*}-C_{u}^{+}$correspondra aux extensions à réaliser sur cet arc en vue d'obtenir la nouvelle valeur du flot maximal.

\section{MO DÈLE NON-LIN ÉAIRE D'EXTEN SION DU RÉSEAU}

L'extension du réseau peut être interprétée comme la définition des décisions diachroniques et synchroniques permettant de faire passer le système à un niveau supérieur d'homéostasie. En conséquence, les outils méthodologiques 
à mettre en œuvre ne peuvent être que d'orientation systémique. L'arsenal de la théorie des graphes peut être mis à profit pour définir un schéma d'extension du réseau permettant d'assurer l'expédition des quantités prévues. Pour cela, des arcs supplémentaires, représentant l'ensemble des solutions envisageables, sont inclus dans le graphe initial. La résolution par le flot maximal permettra d'exclure les décisions non conformes avec les objectifs fixés. Ces décisions se verront affecter un flot nul dans la solution. Cependant, la solution dégagée par la résolution du problème du flot maximal n'est pas toujours unique. C'est le plus souvent une distribution de flux compatibles. D'autres variantes de distribution des flux peuvent donner la même valeur de flot maximal. La détermination d'une solution unique par la mise à profit des algorithmes classiques du flot maximal à coût minimum suppose des fonctions de coûts linéaires ou linéarisables par morceaux [8, 10, 12]. Ces conditions ne sont pas remplies dans le cas du problème traité ici, en raison principalement :

- de la non-linéarité des coûts de contamination et des coûts d'énergie ;

- de la non-connaissance préalable des lignes où le batching sera effectué.

En conséquence, nous nous orienterons vers une solution par programmation non-linéaire. Il s'agira de mettre au point un fonctionnel où apparaîtront les variables de décision suivantes :

- les quantités de brut et de condensat à faire transiter par chaque ligne $G_{B i}$ et $G_{C i}$;

- les stockages supplémentaires à construire et leur localisation.

Le fonctionnel doit également minimiser les coûts totaux représentés par la somme :

$\Psi=\left\{\sum_{i=1}^{n} C_{i}^{\mathrm{cont}}+\sum_{i=1}^{n} C_{i}^{E}+\sum_{j=1}^{m} C_{j}^{R}+\sum_{k=1}^{s} C_{k}^{\mathrm{int}}+\sum_{l=1}^{p} C_{l}^{L}\right\} \Rightarrow \min$

avec :

$C_{i}^{\text {cont }}$ coûts de contamination sur la ligne $i$

$C_{l}^{L} \quad$ coûts de construction de la nouvelle ligne $l$

$C_{j}^{R} \quad$ coûts de construction des stockages supplémentaires au terminal $j$

$C_{k}^{\text {int }} \quad$ coûts de construction des stockages intermédiaires $k$

$C_{i}^{E} \quad$ coûts d'énergie sur la ligne $i$

Le fonctionnel est soumis à quatre types d'ensembles de contraintes :

- l'ensemble des contraintes de conservation des flux :

$$
\begin{aligned}
& \sum_{u \in d^{+}} G_{B u}=\sum_{u \in d^{-}} G_{B u} \\
& \sum_{u \in d^{+}} G_{C u}=\sum_{u \in d^{-}} G_{C u}
\end{aligned}
$$

- les contraintes liées à l'alimentation des raffineries :

$$
G_{B i} \geq V_{i}^{\mathrm{raf}}
$$

- les contraintes de délimitation des capacités des lignes :

$$
G_{B i}+G_{C i} \leq R_{i} G_{i}^{\mathrm{nom}}
$$

- les contraintes de non-négativité :

$$
G_{B i} \geq 0 ; G_{C i} \geq 0 ; d_{i} \geq 0
$$

La formulation définitive du système de contraintes dépend du cas concret à analyser. La solution du modèle devra définir :

- le tracé des nouvelles lignes à construire et leur diamètre ;

- le schéma optimal de transport, autrement dit les lignes que l'on destinera au transport par batch et celles où l'on effectuera le transport d'un seul produit ;

- la localisation et la quantification des capacités supplémentaires de stockage à construire.

Pour cela, on reconduira les hypothèses suivantes :

- le pompage par batch, là où il est présent, se fera avec bouchon de condensat ;

- les capacités nécessaires au stockage du brut devront être supérieures ou égales à l'équivalent de sept jours de pompage.

\section{1 Évaluation des coûts de contamination}

Le batching n'a pas que des avantages. On désigne par le terme de contamination le phénomène de mélange qui se produit au contact de deux cargaisons différentes. Le produit résultant de la contamination est appelé contaminat ou interface. Le phénomène de contamination est le résultat de la diffusion d'un produit dans l'autre. De nombreux travaux expérimentaux ont été menés dans le but de prédire l'importance du contaminat et de déterminer les facteurs favorisant l'accroissement du phénomène [19-24]. Ces travaux ont permis d'établir des formules et des courbes empiriques permettant une prévision correcte du volume de contaminat. De nombreuses relations associent le volume de contaminat à la distance parcourue [24]. Elles sont de la forme :

$$
l=k L^{n}
$$

avec :

$l$ longueur du bouchon de contaminat en mètres

$L \quad$ longueur totale de la conduite en mètres

$k$ et $n$ constantes dépendant de la nature des produits pompés

Ainsi, pour la contamination pétrole-essence, il a été proposé la relation suivante :

$$
l=1,1 L^{0,529}
$$


Des études sur la contamination pétrole-condensat sur la ligne HEH-Bejaia ont montré que la relation (27) pouvait être utilisée avec une erreur inférieure à $8 \%$ [22]. Le volume de contaminat dans un cycle brut-condensat peut être alors déterminé par la relation :

$$
V_{i}^{\text {cycle }}=1,727 d_{1}^{2} L_{i}^{0,529}
$$

Le volume de contaminat sur une année d'exploitation est alors :

$$
V_{i}^{\text {an }}=V_{i}^{\text {cycle }} N_{C i}
$$

avec :

$d_{i} \quad$ diamètre intérieur moyen de l'oléoduc $i$

$L_{i} \quad$ longueur totale de la ligne $i$

$N_{C i}$ nombre de cycles par an sur la ligne $i$ :

$$
N_{C i}=\alpha_{0} \frac{G_{C i}}{R_{C i}}=\alpha_{0} \frac{G_{C i}}{R_{i}^{\text {disp }}+R_{i}^{\text {sup }}-R_{B i}}
$$

$\alpha_{0} \quad$ coefficient tenant compte du stock de sécurité et du remplissage incomplet des réservoirs

$R_{C i}$ capacité de stockage destinée au condensat au terminal $i$

$R_{i}^{\text {disp }}$ capacité de stockage totale disponible au terminal $i$

$R_{i}^{\text {sup }}$ capacité de stockage supplémentaire à construire au terminal $i$

$R_{B i} \quad$ capacité nécessaire au stockage du brut au terminal $i$

En tenant compte de l'hypothèse sur le stockage du brut, les coûts annuels de contamination s'expriment par la relation suivante :

$$
C_{i}^{\text {cont }}=A_{i} \frac{G_{C i}}{R_{i}^{\text {disp }}+R_{i}^{\text {sup }}-0,02 G_{B i}} d_{i}^{2}
$$

avec :

$$
A_{i}=\frac{1}{2}\left(S_{C}-S_{B}\right) 1,727 \alpha_{0} L_{i}^{0,529}
$$

et $S_{C}$, prix du $\mathrm{m}^{3}$ de condensat et $S_{B}$, prix du $\mathrm{m}^{3}$ de brut.

La relation (31) exprime les coûts de contamination en fonction de toutes les variables de décision.

\subsection{Coûts de construction des stockages supplémentaires}

La construction de stockages supplémentaires peut concerner tout aussi bien les terminaux que les stockages intermédiaires. Par stockages intermédiaires, il faut comprendre ici les stockages destinés à assurer l'alimentation ininterrompue des raffineries soutirant du brut d'un point intermédiaire du tracé de la ligne (fig. 8). Lors du passage du bouchon de condensat, l'alimentation de la raffinerie en pétrole brut est assurée à partir du stockage intermédiaire. En considérant que l'alimentation de la raffinerie se fait avec un débit continu $q_{1}$, le volume maximal du bouchon de condensat s'exprime par:

$$
V_{B C}=q_{0} \frac{\alpha_{1} R^{\text {int }}}{q_{l}}
$$

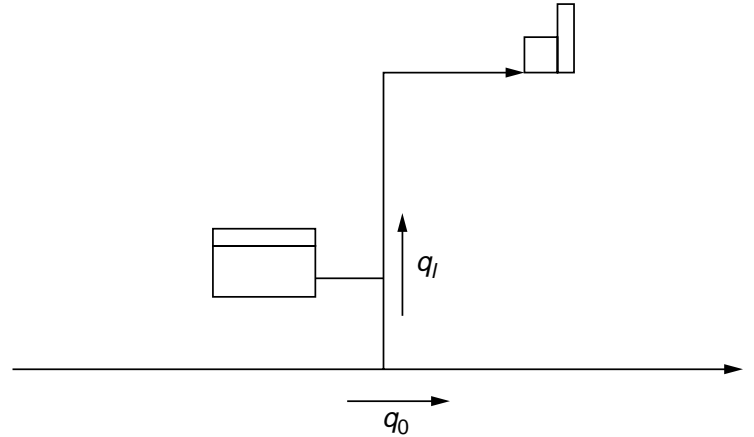

Figure 8

Stockage intermédiaire.

Intermediate storage.

avec $R^{\text {int }}$, capacité de stockage intermédiaire et $\alpha_{1}$, coefficient de sécurité.

Posons :

$$
\varepsilon=\frac{q_{i}}{q_{0}}
$$

et :

$$
R^{\mathrm{int}}=R_{\mathrm{disp}}^{\mathrm{int}}+R_{\mathrm{const}}^{\mathrm{int}}
$$

avec $R_{\text {disp }}^{\text {int }}$, volume de stockage disponible et $R_{\text {const }}^{\text {int }}$, volume de stockage intermédiaire à construire.

Le nombre de cycles de pompage par batch, en tenant compte des limitations imposées par les stockages intermédiaires, s'écrit comme suit :

$$
N_{C}^{\prime}=\frac{G_{C}}{V_{B C}}=\frac{G_{C} \varepsilon}{\alpha_{1}\left(R_{\mathrm{disp}}^{\mathrm{int}}+R_{\text {const }}^{\mathrm{int}}\right)}
$$

Comme on doit avoir obligatoirement $N_{\dot{C}}^{\prime}=N_{C}$, il ressort que le volume de stockage intermédiaire à construire peut s'exprimer comme suit :

$$
R_{\mathrm{const}}^{\mathrm{int}}=\frac{G_{C} \varepsilon}{\alpha_{1} N_{C}}-R_{\mathrm{disp}}^{\mathrm{int}}
$$

D'où, en admettant une durée d'amortissement de dix ans, l'expression des coûts de construction de stockages supplémentaires intermédiaires :

$$
C^{\mathrm{int}}=\frac{S_{R}}{10}\left(\frac{G_{C} \varepsilon}{\alpha_{1} N_{C}}-R_{\mathrm{disp}}^{\mathrm{int}}\right)
$$

avec $S_{R}$, coût de construction d'un stockage de $1 \mathrm{~m}^{3}$.

De la même manière, les coûts de construction de stockages supplémentaires au niveau du terminal $j$ sont donnés par :

$$
C_{j}^{R}=\frac{S_{R}}{10} R_{j}^{\text {sup }}
$$


Pour le parc de stockage départ :

$$
C_{k}^{\mathrm{dep}}=\frac{S_{R}}{10}\left[R_{\mathrm{disp}}^{\mathrm{dep}}+R_{\mathrm{const}}^{\mathrm{dep}}-0,02\left(\sum_{i=1}^{n} G_{B i}+\sum_{i=1}^{n} G_{C i}\right)\right]
$$

Au stade de l'avant-projet, l'estimation des coûts réduits d'une nouvelle ligne peut être établie par l'intermédiaire de la relation $[25]$ :

$$
C^{L}=\frac{k_{0}}{10} d L
$$

où $d$ est le diamètre de la conduite en pouces et $L$, la longueur de la conduite en mètres.

\subsection{Coûts d'énergie lors du pompage par batch}

Les propriétés physico-chimiques du pétrole brut et du condensat sont assez différentes. Le condensat est plus léger et surtout nettement moins visqueux que le brut. En conséquence, les coûts d'énergie varient d'une manière significative en fonction de l'importance des volumes transportés par batch. La puissance de pompage nécessaire au transport des colonnes de brut durant une année s'exprime par:

$$
W_{1}=\frac{1}{\eta}\left(\rho_{1} g h_{1} q_{m}\right) T_{\text {brut }}^{\text {an }}
$$

Pour le condensat :

$$
W_{2}=\frac{1}{\eta}\left(\rho_{2} g h_{2} q_{m}\right) T_{\text {cond }}^{\mathrm{an}}
$$

avec :

$h_{1}$ et $h_{2} \quad$ respectivement les pertes de charge engendrées par la colonne de brut et celles provoquées par le bouchon de condensat

$\rho_{1}$ et $\rho_{2} \quad$ densités du brut et du condensat

$q_{m} \quad$ débit moyen de la ligne en $\mathrm{m}^{3} / \mathrm{h}$

$T_{\text {brut }}^{\text {an }} \quad$ temps de pompage du brut et du condensat durant

et $T_{\text {cond }}^{\text {an }} \quad$ une année

$\eta \quad$ rendement global de la ligne

En admettant un écoulement turbulent dans la zone des tubes hydrauliquement lisses, on peut écrire :

$$
\begin{aligned}
& h_{1}=\frac{\beta q_{m}^{1,75} \gamma_{1}^{0,25}}{d^{4,75}} L \\
& h_{2}=\frac{\beta q_{m}^{1,75} \gamma_{2}^{0,25}}{d^{4,75}} L
\end{aligned}
$$

avec :

$\gamma_{1}$ et $\gamma_{2} \quad$ viscosités du brut et du condensat $\beta \quad$ constante caractérisant l'écoulement

Let $d \quad$ longueur et diamètre moyen intérieur de la conduite

Or :

$$
T_{\mathrm{brut}}^{\mathrm{an}}=\frac{G_{B}}{q_{m}}
$$

$$
T_{\text {cond }}^{\text {an }}=\frac{G_{C}}{q_{m}}
$$

De plus, sachant qu'un oléoduc fonctionne en moyenne 8400 heures par an, on peut écrire :

$$
q_{m}=\frac{G_{C}+G_{B}}{8400}
$$

Les coûts d'énergie s'expriment alors sous la forme :

$$
C^{E}=S_{E} \frac{k}{d^{4,75}}\left(G_{B}+G_{C}\right)^{1,75}\left(\rho_{1} \gamma_{1}^{0,25} G_{B}+\rho_{2} \gamma_{2}^{0,25} G_{c}\right)
$$

où $S_{E}$ est le coût du kilowatt/heure d'énergie, et :

$$
k=\frac{\beta \mathrm{g} L}{\eta(8400)^{1,75}}
$$

\subsection{Algorithme de résolution}

Le problème ainsi posé se ramène donc à un modèle de programmation non-linéaire multivariable. Il est à remarquer que l'ensemble des contraintes, délimitant le domaine des solutions admissibles, est linéaire. Cette dernière particularité est à la base de la procédure de résolution préconisée. L'idée générale de l'algorithme de Franck-Wolfe consiste à linéariser le fonctionnel en un point donné du domaine des solutions admissibles, ce qui permet de ramener le problème initial à un problème de programmation linéaire multiétape, que l'on résout par une procédure itérative incluant un algorithme de très grande efficacité : l'algorithme du simplexe. Cependant pour la détermination de l'optimum global, en raison de la non-convexité de la fonction de but, le problème doit être résolu pour différentes valeurs du point initial. On utilise habituellement dans ce cas deux approches $[3,26]$ :

- des techniques basées sur le fractionnement du domaine des solutions (Search Clustering Approach) où la résolution est recherchée pour tous les sous-domaines avant d'être comparée ;

- des techniques de génération aléatoire du point initial (Random Search Approach) basées sur l'usage d'une fonction de densité de probabilité à variance fixée heuristiquement.

La première démarche se démarque par la simplicité de sa mise en œuvre mais également par un temps machine croissant de manière exponentielle en fonction du nombre de variables. Ainsi, pour un problème de 14 variables, la division par seulement 4 de l'intervalle de variation de chaque variable se traduit par la résolution de 268435456 problèmes locaux d'optimisation. La seconde approche, particulièrement dans sa version ARS (Adaptive Random Search), est nettement plus rapide que la première mais nécessite aussi un temps machine relativement important. Pour contourner ce problème, en liaison avec la structure particulière du modèle, le point initial est choisi dans 
l'ensemble réduit des solutions compatibles dégagées par la résolution du problème du flot maximal, ce qui nous ramène, pour le cas d'un réseau de l'envergure du réseau algérien, à la résolution d'une dizaine au plus de problèmes locaux d'optimisation.

Sous forme compacte, le problème peut être présenté comme suit :

$$
\begin{gathered}
\Phi(\stackrel{\mathrm{r}}{X}) \Rightarrow \min \\
g_{j}\left(X^{\mathrm{I}}\right) \leq 0 \\
x_{i} \geq 0 \\
X=\left\{x_{1} ; x_{2} ; \ldots ; x_{n}\right\}
\end{gathered}
$$

La démarche à suivre est la suivante :

- circonscrire l'ensemble des solutions compatibles ;

- choisir un point initial appartenant au domaine des solutions compatibles $\dot{X}^{(k)}$;

- linéariser le fonctionnel au point $\dot{X}^{(k)}$. Pour cela, déterminer la valeur du gradient :

$$
\nabla \Phi\left(\stackrel{r}{X}^{(k)}\right)=\left\{\frac{\partial \Phi\left(\stackrel{\mathfrak{X}}{ }^{(k)}\right)}{\partial x_{1}} ; \frac{\partial \Phi\left(\stackrel{\mathfrak{l}}{ }^{(k)}\right)}{\partial x_{2}} ; \ldots ; \frac{\partial \Phi\left(\stackrel{\mathrm{I}}{X}^{(k)}\right)}{\partial x_{n}}\right\}
$$

- approximer le fonctionnel au point $\dot{X}^{(k)}$ par la relation linéaire suivante :

$$
F=\sum_{i=1}^{n} \frac{\partial \Phi\left(\stackrel{I}{X}^{(k)}\right)}{\partial x_{i}} x_{i}
$$

- soumettre le fonctionnel à l'ensemble des contraintes linéaires $g_{i}(\dot{X})$ et résoudre par le simplexe ;

- soit $\dot{Z}^{(k)}$ la solution du sous-problème linéaire à l'étape $k$. Le point d'itération suivant est déterminé par la relation :

$$
\begin{gathered}
\stackrel{r}{X}^{(k+1)}=\stackrel{r}{X}^{(k)}+\alpha_{k}\left(\stackrel{r}{Z}^{(k)}-\stackrel{r}{X}^{(k)}\right) \\
0<\alpha_{k}<1
\end{gathered}
$$

- effectuer le test de cessation des itérations, qui peut être de la forme :

$$
\left|\Phi\left(\stackrel{r}{X}^{(k+1)}\right)-\Phi\left(\stackrel{r}{X}^{(k)}\right)\right| \leq \varepsilon
$$

- si le test est vérifié, $\dot{X}^{(k+1)}$ est admis comme solution provisoire et l'on reprend la procédure pour un nouveau point initial ; sinon, on effectue une nouvelle itération en linéarisant le fonctionnel au point $\dot{X}^{(k+1)}$.

\section{APPLCATION AU RÉSEAU ALGÉRIEN DE TRAN SPO RT DE BRUT ET DE CON DEN SAT}

\section{1 Évaluation des capacités du réseau}

L'application des modèles est effectuée sur un exemple s'inspirant très largement du réseau algérien de transport de brut et de condensat. La théorie des graphes et la mise à profit du problème du flot maximal permettent d'apporter des éléments de réponse aux questions relatives :

- aux possibilités du réseau, dans sa configuration actuelle, à assurer l'expédition des prévisions de production à l'horizon 2003 (tableau 4);

TABLEAU 4

Prévisions de production de brut et de condensat pour 2003

Estimates of crude and condensate production for 2003

\begin{tabular}{l|c|c}
\hline Zones & Brut (t/an) & Condensat (t/an) \\
\hline Haoud El Hamra & 60000000 & 8200000 \\
Hassi Rmel & 2000000 & 10000000 \\
In Amenas & 2500000 & 600000 \\
\hline Total & 64500000 & 18800000 \\
\hline
\end{tabular}

- à l'incidence des décisions synchroniques de réaffectation des flux, par la généralisation du pompage par bouchon, sur la capacité totale du réseau, la sécurité d'alimentation des raffineries et les risques de saturation des ports d'expédition.

Dans les graphes servant de modèle au réseau, chaque arc dispose de deux valeurs : une borne inférieure $C_{u}^{-}$(le plus souvent égale à zéro), et une borne supérieure $C_{u}^{+}$ correspondant à la capacité maximale de l'arc. On notera que dans ce paragraphe, les capacités maximales sont supposées équivalentes aux capacités nominales. Ceci correspond à un scénario incluant la réhabilitation de toutes les lignes du réseau. Par réhabilitation, on entend l'exécution de différentes opérations destinées à ramener la capacité réelle d'une ligne à sa capacité nominale. Ces opérations incluent principalement $[27,28]$ :

- le ramonage de la conduite ;

- le diagnostic par outil « intelligent»;

- la réfection des tronçons corrodés (norme B31.G);

- la rénovation des stations de pompage.

L'alimentation des raffineries étant prioritaire, les arcs qui lui correspondent verront leurs deux bornes affectées d'une valeur unique équivalente à leur demande. La borne supérieure de l'arc puits, inconnue à l'avance, est affectée d'un nombre très grand $(M=200$ millions de tonnes/an dans notre exemple).

La réponse à la première question, relative aux possibilités du réseau dans sa configuration actuelle, découle de l'analyse 
TABLEAU 5

Flot maximal dans la configuration actuelle Maximal flow in current functioning

\begin{tabular}{|c|c|c|c|c|c|}
\hline Désignation & Arc & $C_{u}^{-}$ & $C_{u}^{+}$ & Flux & Couleur \\
\hline Source condensat & $(s, 1)$ & 0 & $M$ & 12200 & rouge \\
\hline Source brut & $(s, 2)$ & 0 & $M$ & 37700 & rouge \\
\hline Production condensat HEH & $(1,4)$ & 0 & 8300 & 3800 & rouge \\
\hline Production condensat HRM & $(1,15)$ & 0 & 10000 & 8400 & rouge \\
\hline Production brut $\mathrm{HEH}$ & $(2,3)$ & 0 & 60000 & 33200 & rouge \\
\hline Production brut INAS & $(2,28)$ & 0 & 2500 & 2500 & vert \\
\hline Production brut HRM & $(2,36)$ & 0 & 2000 & 2000 & vert \\
\hline Stockage brut HEH & $(3,6)$ & 0 & 33200 & 33200 & vert \\
\hline Stockage condensat HEH & $(4,5)$ & 0 & 3800 & 3800 & vert \\
\hline Ligne condensat HEH-Bejaia & $(5,9)$ & 0 & 17000 & 3800 & rouge \\
\hline Ligne condensat HEH-HRM & $(5,15)$ & 0 & 700 & 0 & noir \\
\hline Ligne brut HEH-piquage OB1 & $(6,7)$ & 0 & 17000 & 2800 & rouge \\
\hline Ligne brut HEH-piquage OZ1 & $(6,38)$ & 0 & 21600 & 800 & rouge \\
\hline Ligne brut HEH-Skikda & $(6,23)$ & 0 & 30000 & 29600 & rouge \\
\hline Ligne brut piquage OB1-Bejaia & $(7,8)$ & 0 & 17000 & 0 & noir \\
\hline Alimentation raffinerie d'Alger & $(7,35)$ & 2800 & 2800 & 2800 & noir \\
\hline Stockage condensat Bejaia & $(9,10)$ & 0 & 8200 & 3800 & rouge \\
\hline Stockage brut Bejaia & $(8,10)$ & 0 & 15800 & 0 & noir \\
\hline Arc déconnectant & $(10,11)$ & 0 & 17000 & 3800 & noir \\
\hline Poste de chargement 1 Bejaia & $(11,12)$ & 0 & 12000 & 3800 & rouge \\
\hline Poste de chargement 2 Bejaia & $(11,13)$ & 0 & 8000 & 0 & noir \\
\hline Poste de chargement 3 Bejaia & $(11,14)$ & 0 & 6000 & 0 & noir \\
\hline Stockage condensat HRM & $(15,16)$ & 0 & 8400 & 8400 & vert \\
\hline Ligne condensat HRM-Arzew & $(16,17)$ & 0 & 21000 & 8400 & rouge \\
\hline Stockage condensat Arzew & $(17,18)$ & 0 & 19000 & 8400 & rouge \\
\hline Poste de chargement 1 Arzew & $(18,19)$ & 0 & 16000 & 400 & rouge \\
\hline Poste de chargement 2 Arzew & $(18,20)$ & 0 & 10000 & 0 & noir \\
\hline Poste de chargement 3 Arzew & $(18,21)$ & 0 & 8000 & 8000 & vert \\
\hline Ligne brut piquage OZ1-Arzew & $(38,22)$ & 0 & 21600 & 2800 & rouge \\
\hline Stockage brut Arzew & $(22,18)$ & 0 & 15000 & 0 & noir \\
\hline Alimentation raffinerie d'Arzew & $(22,35)$ & 2800 & 2800 & 2800 & noir \\
\hline Stockage brut INAS & $(28,29)$ & 0 & 7200 & 2500 & rouge \\
\hline Stockage brut HRM & $(36,37)$ & 0 & 2000 & 2000 & vert \\
\hline Ligne brut piquage HRM-piquage OZ1 & $(37,38)$ & 0 & 2500 & 2000 & rouge \\
\hline Ligne brut INAS-HEH & $(29,3)$ & 0 & 18000 & 0 & noir \\
\hline Ligne brut INAS-Skirra & $(29,30)$ & 0 & 7800 & 2500 & rouge \\
\hline Stockage brut Skirra & $(30,31)$ & 0 & 10000 & 2500 & rouge \\
\hline Poste de chargement 1 Skirra & $(31,32)$ & 0 & 8000 & 2500 & rouge \\
\hline Poste de chargement 2 Skirra & $(31,33)$ & 0 & 6000 & 0 & noir \\
\hline Poste de chargement 3 Skirra & $(31,34)$ & 0 & 4000 & 0 & noir \\
\hline Alimentation raffinerie de Skikda & $(24,35)$ & 15700 & 15700 & 15700 & noir \\
\hline Poste de chargement 1 Skikda & $(24,25)$ & 0 & 16000 & 5900 & rouge \\
\hline Poste de chargement 2 Skikda & $(24,26)$ & 0 & 12000 & 0 & noir \\
\hline Poste de chargement 3 Skikda & $(24,27)$ & 0 & 8000 & 8000 & vert \\
\hline Stockage brut Skikda & $(23,24)$ & 0 & 30000 & 29600 & rouge \\
\hline
\end{tabular}

Flot max : 49900 milliers de tonnes/an 
des résultats du scénario de base : batching sur la ligne HEHBejaia uniquement et pompage du brut sur les autres lignes. Il ressort que (tableau 5) :

- le réseau ne peut assurer l'expédition de la totalité des prévisions ( $\varphi_{0}=49,9$ MTA) ;

- seules 37,7 millions de tonnes de brut et 12,2 millions de tonnes de condensat pourraient être livrées, soit $47 \%$ de la production de la zone de HEH, $87 \%$ de celle de Hassi Rmel et $81 \%$ de prévisions pour In Amenas ;

- l'expédition des quantités de condensat produites au niveau d'In Amenas n'est pas assurée.

Le scénario incluant la possibilité de réalisation du pompage par bouchon sur d'autres lignes se traduit par un nouveau graphe (fig. 9). La solution par le flot maximal définira automatiquement les chaînes où ce procédé sera efficace et celles qui devront n'assurer le pompage que d'un seul produit. L'analyse des résultats de cette deuxième variante (tableau 6) montre que :

- même dans ce contexte, le réseau ne pourra pas assurer l'expédition des quantités prévues ;

- le flot maximal est légèrement amélioré par rapport au scénario de base $\left(\varphi_{0}=50,5\right.$ MTA $)$;

- ce schéma de pompage permet l'évacuation de 37,7 millions de tonnes de brut et de 12,8 millions de tonnes de condensat par an ;

- le batching est effectué sur deux lignes : HEH-Skikda et INAS-Skirra ;

- la totalité de la production de condensat d'In Amenas est expédiée par batching sur la ligne INAS-Skirra ;

- la demande des raffineries nationales reste assurée ;

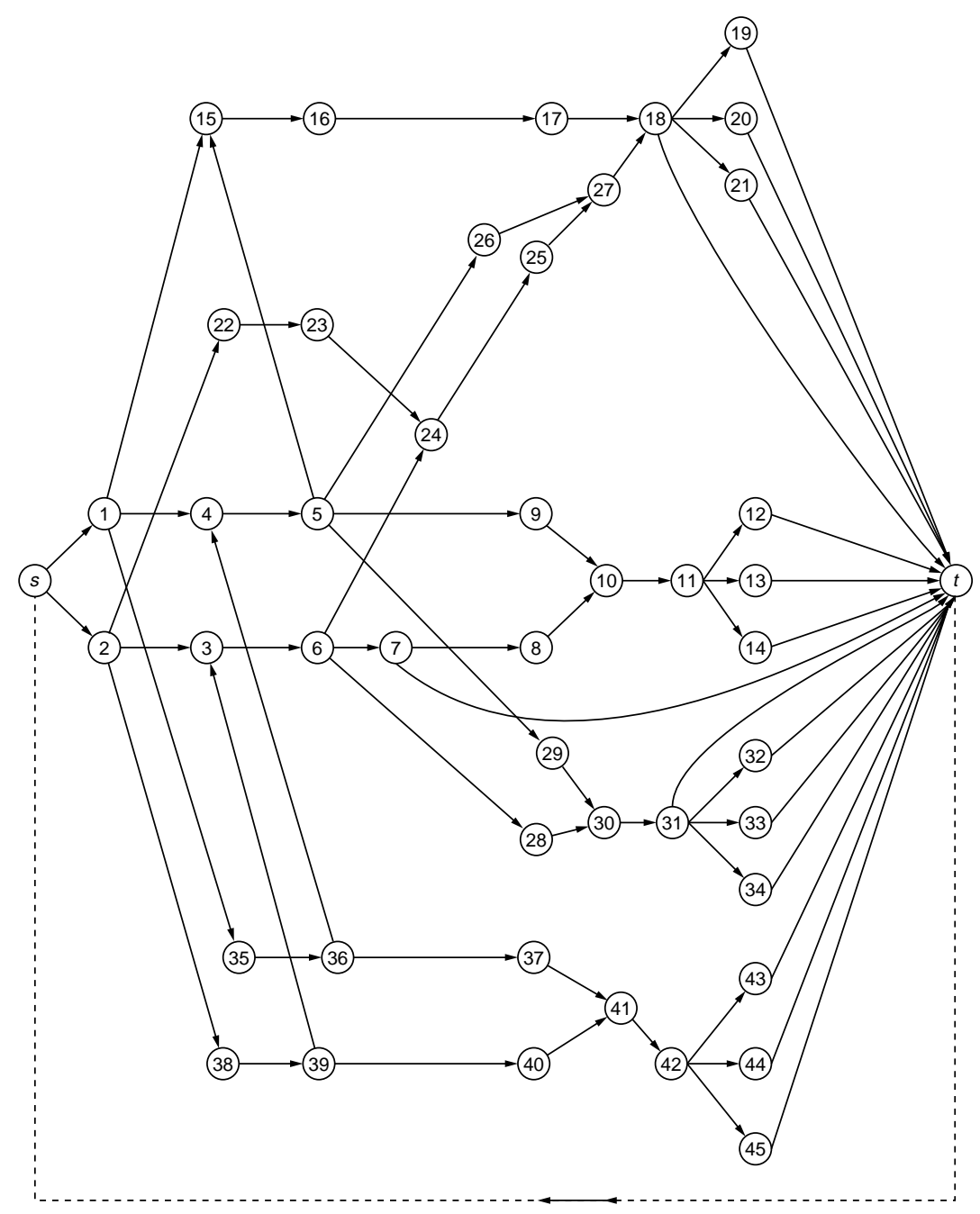

Figure 9

Graphe du réseau algérien avec batching sur plusieurs lignes.

Graph of the Algerian network with generalization of the batching. 
TABLEAU 6

Flot maximal avec batching sur plusieurs lignes

Maximal flow with generalization of the batching

\begin{tabular}{|c|c|c|c|c|c|}
\hline Désignation & Arc & $C_{u}^{-}$ & $C_{u}^{+}$ & Flux & Couleur \\
\hline Source condensat & $(s, 1)$ & 0 & $\mathrm{M}$ & 12800 & noir \\
\hline Source brut & $(s, 2)$ & 0 & M & 37700 & noir \\
\hline Production condensat HRM & $(1,15)$ & 0 & 10100 & 8400 & rouge \\
\hline Production condensat HEH & $(1,4)$ & 0 & 8300 & 3800 & rouge \\
\hline Production condensat INAS & $(1,35)$ & 0 & 600 & 600 & vert \\
\hline Production brut HRM & $(2,22)$ & 0 & 2000 & 2000 & vert \\
\hline Production brut HEH & $(2,3)$ & 0 & 60000 & 33200 & rouge \\
\hline Production brut INAS & $(2,38)$ & 0 & 2500 & 2500 & vert \\
\hline Stockage condensat HRM & $(15,16)$ & 0 & 8400 & 8400 & vert \\
\hline Stockage condensat HEH & $(4,5)$ & 0 & 3800 & 3800 & vert \\
\hline Stockage condensat INAS & $(35,36)$ & 0 & 4500 & 600 & rouge \\
\hline Stockage brut HRM & $(22,23)$ & 0 & 2000 & 2000 & vert \\
\hline Stockage brut HEH & $(3,6)$ & 0 & 33200 & 33200 & vert \\
\hline Stockage brut INAS & $(38,39)$ & 0 & 7200 & 2500 & rouge \\
\hline Ligne condensat HRM-Arzew & $(16,17)$ & 0 & 21000 & 8400 & rouge \\
\hline Stockage condensat Arzew 1 & $(17,18)$ & 0 & 19000 & 8400 & rouge \\
\hline Alimentation raffinnerie d'Arzew & $(18,46)$ & 2800 & 2800 & 2800 & noir \\
\hline Poste de chargement 1 Arzew & $(18,20)$ & 0 & 16000 & 11800 & rouge \\
\hline Poste de chargement 2 Arzew & $(18,21)$ & 0 & 10000 & 0 & noir \\
\hline Poste de chargement 3 Arzew & $(18,22)$ & 0 & 8000 & 0 & noir \\
\hline Ligne condensat HEH-HRM & $(5,15)$ & 0 & 700 & 0 & noir \\
\hline Ligne condensat HEH-Bejaia & $(5,9)$ & 0 & 17000 & 0 & noir \\
\hline Ligne condensat HEH-Arzew & $(5,26)$ & 0 & 21000 & 0 & noir \\
\hline Ligne condensat HEH-Skikda & $(5,29)$ & 0 & 30000 & 3800 & rouge \\
\hline Stockage condensat Bejaia & $(9,10)$ & 0 & 8200 & 0 & noir \\
\hline Arc fictif & $(10,11)$ & 0 & 17000 & 0 & noir \\
\hline Ligne condensat INAS-HEH & $(36,4)$ & 0 & 9000 & 0 & noir \\
\hline Ligne condensat INAS-Skirra & $(36,37)$ & 0 & 7800 & 600 & rouge \\
\hline Stockage condensat Skirra & $(37,41)$ & 0 & 5000 & 600 & rouge \\
\hline Arc fictif & $(41,42)$ & 0 & 7800 & 3100 & noir \\
\hline Stockage condensat Arzew 2 & $(26,27)$ & 0 & 15000 & 0 & noir \\
\hline Arc fictif & $(27,18)$ & 0 & 15000 & 6200 & noir \\
\hline Stockage condensat Skikda & $(29,30)$ & 0 & 30000 & 3800 & rouge \\
\hline Arc fictif & $(30,31)$ & 0 & 30000 & 30000 & noir \\
\hline Ligne brut HRM-piquage OZ1 & $(23,24)$ & 0 & 2500 & 2000 & rouge \\
\hline Ligne brut piquage OZ1-Arzew & $(24,25)$ & 0 & 21600 & 6200 & rouge \\
\hline Ligne brut HEH-piquage OZ1 & $(6,24)$ & 0 & 21600 & 4200 & rouge \\
\hline Ligne brut HEH-piquage OB1 & $(6,7)$ & 0 & 17000 & 2800 & rouge \\
\hline Ligne brut HEH-Skikda & $(6,28)$ & 0 & 30000 & 26200 & rouge \\
\hline Ligne brut piquage OB1-Bejaia & $(7,8)$ & 0 & 17000 & 0 & noir \\
\hline Alimentation raffinerie d'Alger & $(7,46)$ & 0 & 2800 & 2800 & noir \\
\hline Stockage brut Bejaia & $(8,10)$ & 0 & 15800 & 0 & noir \\
\hline Stockage brut Arzew & $(25,27)$ & 0 & 15000 & 6200 & rouge \\
\hline Stockage brut Skikda & $(28,30)$ & 0 & 30000 & 26200 & rouge \\
\hline Ligne brut INAS-HEH & $(39,3)$ & 0 & 9000 & 0 & noir \\
\hline Ligne brut INAS-Skirra & $(39,40)$ & 0 & 7800 & 2500 & rouge \\
\hline Stockage brut Skirra & $(40,41)$ & 0 & 5000 & 2500 & rouge \\
\hline Alimentation raffinerie de Skikda & $(31,46)$ & 15700 & 15700 & 15700 & noir \\
\hline Poste de chargement 1 Skikda & $(31,32)$ & 0 & 16000 & 6300 & rouge \\
\hline Poste de chargement 2 Skikda & $(31,33)$ & 0 & 12000 & 0 & noir \\
\hline Poste de chargement 3 Skikda & $(31,34)$ & 0 & 8000 & 8000 & vert \\
\hline Poste de chargement 1 Bejaia & $(11,12)$ & 0 & 12000 & 0 & noir \\
\hline Poste de chargement 2 Bejaia & $(11,13)$ & 0 & 8000 & 0 & noir \\
\hline Poste de chargement 3 Bejaia & $(11,14)$ & 0 & 6000 & 0 & noir \\
\hline Poste de chargement 1 Skirra & $(46,43)$ & 0 & 8000 & 3100 & rouge \\
\hline Poste de chargement 2 Skirra & $(46,44)$ & 0 & 6000 & 0 & noir \\
\hline Poste de chargement 3 Skirra & $(46,45)$ & 0 & 4000 & 0 & noir \\
\hline
\end{tabular}

Flot max : 50500 milliers de tonnes/an 
TABLEAU 7

Flot maximal pour différentes variantes de simulation

Maximal flow for different simulations

\begin{tabular}{|c|c|c|c|c|}
\hline \multicolumn{2}{|c|}{ Variantes de simulation } & \multirow{2}{*}{$\begin{array}{c}\varphi_{0} \\
50,5\end{array}$} & \multirow{2}{*}{$\begin{array}{c}\begin{array}{c}\text { Brut } \\
\text { (MTA) }\end{array} \\
37,7\end{array}$} & \multirow{2}{*}{$\begin{array}{c}\begin{array}{c}\text { Condensat } \\
\text { (MTA) }\end{array} \\
12,8\end{array}$} \\
\hline 1 & Scénario de base & & & \\
\hline 2 & Rénovation des SP de la ligne HEH-Bejaia & 50,5 & 37,7 & 12,8 \\
\hline 3 & Réhabilitation de la ligne HEH-Skikda & 50,5 & 37,7 & 12,8 \\
\hline 4 & Construction d'un réservoir de $52000 \mathrm{~m}^{3}$ à HRM & 52,1 & 37,7 & 14,4 \\
\hline 5 & Construction de 12 réservoirs de $52000 \mathrm{~m}^{3}$ à $\mathrm{HEH}$ & 76,3 & 59,1 & 17,2 \\
\hline 6 & Variante combinée A & 81,735 & 62,935 & 18,8 \\
\hline 7 & Variante combinée B & 82,235 & 64,435 & 18,8 \\
\hline
\end{tabular}

- les goulots d'étranglement (arcs verts) se situent au niveau des capacités de stockage disponibles à Haoud El Hamra et Hassi Rmel.

Il est à noter que pour les arcs sources, représentant la production, l'interprétation des couleurs est différente. Les arcs verts désigneront les zones dont la totalité de la production de brut ou de condensat sera évacuée, et les arcs rouges les zones où seule une partie de la production pourra être expédiée.

Il ressort donc de l'analyse de ces résultats que des actions de réhabilitation sans extensions préalables des capacités de stockage aux niveaux de HEH et HRM n'auraient pratiquement aucune incidence sur l'amélioration de la capacité de transit du réseau dans sa globalité.

\subsection{Simulation de décisions synchroniques d'extension des stockages et de réhabilitation}

À l'inverse du paragraphe précédent, on tiendra compte ici de la fiabilité des lignes et l'on procédera à la simulation de différentes variantes d'extension des stockages et de réhabilitation des lignes. On utilisera pour cet objectif le graphe de la figure 9. Les résultats de simulation d'un certain nombre de variantes sont donnés dans le tableau 7.

La variante combinée A suppose les actions simultanées suivantes :

- construction d'un réservoir de $52000 \mathrm{~m}^{3}$ à HRM pour le stockage du brut;

- construction de 10 réservoirs supplémentaires de $52000 \mathrm{~m}^{3}$ pour le stockage du brut à $\mathrm{HEH}$;

- construction de 2 réservoirs de $52000 \mathrm{~m}^{3}$ pour le stockage du condensat à $\mathrm{HEH}$;

- rénovation de toutes les stations de pompage de la ligne HEH-Bejaia ;

- transformation de 2 réservoirs de stockage de brut au niveau d'Arzew pour le stockage du condensat ;
- transformation de 2 réservoirs de stockage de brut au niveau de Skikda pour servir au stockage du condensat ;

- utilisation d'un réservoir de brut au niveau d'INAS pour le stockage du condensat.

La variante combinée B englobe toutes les actions de la variante $\mathrm{A}$, plus la réhabilitation de la ligne $\mathrm{HEH}-\mathrm{Skikda}$.

La même démarche peut être mise à profit pour :

- l'estimation de la flexibilité du réseau et de sa capacité à réagir à toute forme de perturbation ;

- la planification des opérations d'un programme de réhabilitation du réseau. Un tel programme implique l'arrêt volontaire et prolongé de nombreux équipements. Ces arrêts doivent être planifiés de manière à ce que l'incidence sur le flot maximal soit réduite le plus possible.

\subsection{Extension optimale du résea u}

Il ressort des résultats précédents la nécessité de prévoir des opérations de renforcement du réseau en vue de réaliser les objectifs de production à l'horizon 2003. Dans ce contexte, nous userons du modèle non-linéaire d'extension optimale du réseau. Le modèle est appliqué sur un exemple s'inspirant très largement du réseau algérien de transport de brut et de condensat (fig. 10). On procède d'abord par une numérotation artificielle des terminaux de stockage et on désigne les variables par:

$X_{i j} \quad$ quantités annuelles de condensat à faire transiter par la ligne $(i-j)$

$Y_{i j} \quad$ quantités annuelles de brut à faire transiter par la ligne $(i-j)$

$X_{i j}^{\prime} \quad$ quantités de condensat à faire transiter par la nouvelle ligne virtuelle $(i-j)$

$Y_{i j}^{\prime} \quad$ quantités annuelles de brut à faire transiter par la nouvelle ligne virtuelle $(i-j)$

$Z_{k} \quad$ stockages supplémentaires à installer au terminal $k$ 


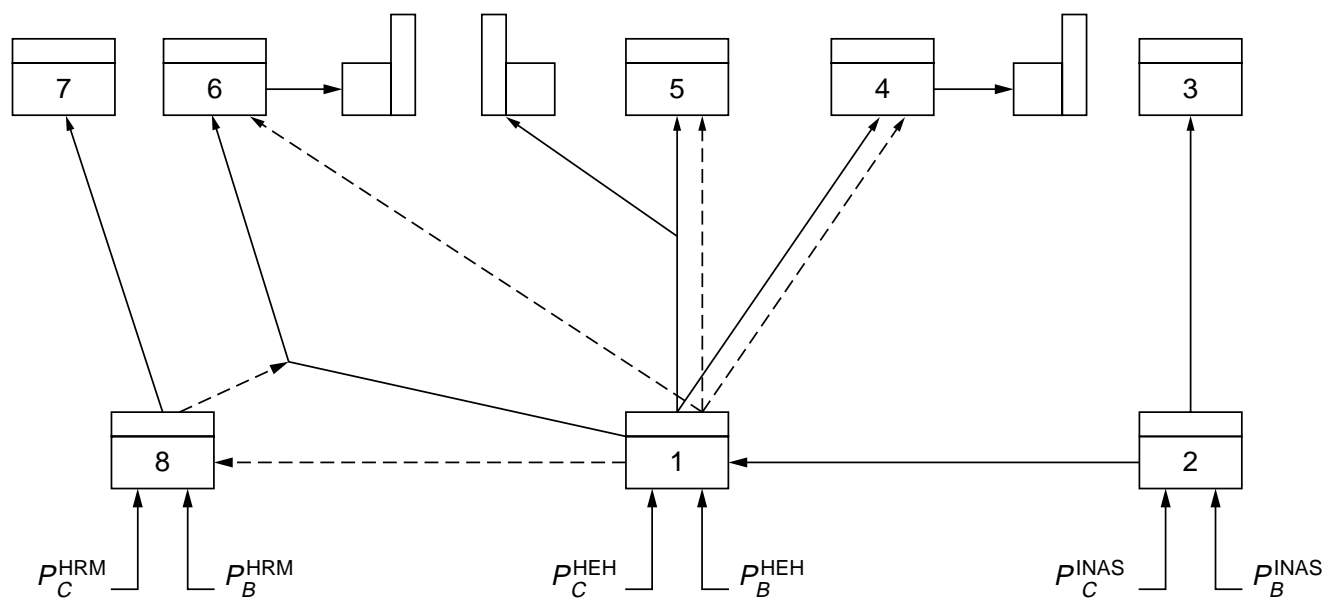

Figure 10

Hypothèses d'extension du réseau.

Hypotheses of a network extension.

$d_{i j} \quad$ diamètre de la nouvelle ligne virtuelle $(i-j)$. Pour les lignes existantes, le diamètre intervient comme une constante

$d_{i j}^{*} \quad$ diamètre normalisé immédiatement supérieur à $d_{i j}$

En relation avec l'exploitation actuelle du réseau, on admettra les hypothèses suivantes :

- la production de brut au niveau de Hassi Rmel, $P_{B}^{\text {HRM }}$ est injectée en totalité dans la ligne (1-6) ;

- la ligne (8-7) est destinée exclusivement au pompage du condensat ;

- les extensions possibles concernent : l'ensemble des parcs de stockage ; la nouvelle ligne (1-8) pour le transport du condensat ; le doublement éventuel des lignes (1-4), (1-5) et (1-6).

Dans ce contexte, le fonctionnel (20) est soumis à l'ensemble des contraintes suivantes, délimitant le domaine des solutions admissibles :

- contraintes d'équilibre des flux : pour le condensat :

$X_{14}+X_{14}^{\prime}+X_{15}+X_{15}^{\prime}+X_{16}+X_{16}^{\prime}+X_{18}^{\prime}-X_{21}=P_{C}^{\mathrm{HEH}}$

$$
\begin{aligned}
& X_{21}+X_{23}=P_{C}^{\mathrm{INAS}} \\
& X_{87}+X_{18}^{\prime}=P_{C}^{\mathrm{HRM}}
\end{aligned}
$$

pour le brut :

$$
Y_{14}+Y_{14}^{\prime}+Y_{15}+Y_{15}^{\prime}+Y_{16}+Y_{16}^{\prime}-Y_{21}=P_{B}^{\mathrm{HEH}}
$$

$$
Y_{21}+Y_{23}=P_{B}^{\mathrm{INAS}}
$$

- contraintes d'alimentation des raffineries :

$$
\begin{aligned}
& Y_{14}+Y_{14}^{\prime} \geq V_{4}^{\text {raf }} \\
& Y_{15}+Y_{15}^{\prime} \geq V_{5}^{\text {raf }} \\
& Y_{16}+Y_{16}^{\prime} \geq V_{6}^{\text {raf }}
\end{aligned}
$$

- contraintes de limitation des capacités de transit des lignes :

$$
X_{14}+Y_{14} \leq R_{14} G_{14}^{\text {nom }}
$$

$$
X_{15}+Y_{15} \leq R_{15} G_{15}^{\text {nom }}
$$

$$
X_{16}+Y_{16}+P_{B}^{\mathrm{HRM}} \leq R_{16} G_{16}^{\mathrm{nom}}
$$

$$
X_{23}+Y_{23} \leq R_{23} G_{23}^{\mathrm{nom}}
$$

$$
X_{21}+Y_{21} \leq R_{21} G_{21}^{\text {nom }}
$$

$$
X_{87} \leq R_{87} G_{87}^{\text {nom }}
$$


TABLEAU 8

Décisions d'extension optimale du réseau

Decisions of network optimal extension

\begin{tabular}{|c|c|c|c|c|c|}
\hline Variables & Unité & $\begin{array}{l}\text { Variante } 1 \\
(R=0,95)\end{array}$ & $\begin{array}{l}\text { Variante } 2 \\
(R=0,92)\end{array}$ & $\begin{array}{l}\text { Variante } 3 \\
(R=0,95)\end{array}$ & $\begin{array}{l}\text { Variante } 4 \\
(R=0,92)\end{array}$ \\
\hline$X_{87}$ & MTA & 18,20 & 18,20 & 18,80 & 18,80 \\
\hline$X_{23}$ & MTA & 0,60 & 0,60 & 0 & 0 \\
\hline$X_{18}$ & MTA & 8,20 & 8,20 & 8,80 & 8,80 \\
\hline$X_{21}$ & MTA & 0 & 0 & 0,60 & 0,60 \\
\hline$Y_{16}$ & MTA & 15,35 & 17 & 16,85 & 17 \\
\hline$Y_{15}$ & MTA & 16,15 & 15,50 & 16,15 & 15,50 \\
\hline$Y_{14}$ & MTA & 28,50 & 27,50 & 28,50 & 27,50 \\
\hline$Y_{23}$ & MTA & 2,5 & 2,50 & 1 & 2,50 \\
\hline$Y_{21}$ & MTA & 0 & 0 & 1,50 & 0 \\
\hline$Z_{1}$ & Milliers de $\mathrm{m}^{3}$ & $623,9(12)$ & $623,9(12)$ & $742,2(14)$ & 717,7 (14) \\
\hline$Z_{8}$ & Milliers de $\mathrm{m}^{3}$ & $194,1(4)$ & $194,1(4)$ & $205,9(4)$ & 205,9 (4) \\
\hline$Z_{7}$ & Milliers de $\mathrm{m}^{3}$ & $31,3(1)$ & $31,3(1)$ & $73(2)$ & $76,4(2)$ \\
\hline$d_{18}^{*}$ & Pouces & 20 & 20 & 20 & 20 \\
\hline
\end{tabular}

Les contraintes de non-négativité sont données par:

$$
\begin{aligned}
& X_{i j} \geq 0 ; X_{i j}^{\prime} \geq 0 \text { pour tous les couples }(i-j) \\
& Y_{i j} \geq 0 ; Y_{i j}^{\prime} \geq 0 \text { pour tous les couples }(i-j)
\end{aligned}
$$

$$
Z_{k} \geq 0 \quad \text { pour les tous } k
$$

$$
d_{i j} \geq 0 \quad \text { pour tous les couples }(i-j)
$$

avec :

$P_{B}^{\mathrm{HEH}}$ et $P_{C}^{\mathrm{HEH}}$ respectivement production de brut et de condensat au niveau de Haoud El Hamra

$P_{B}^{\mathrm{HRM}}$ et $P_{C}^{\mathrm{HRM}}$ production de brut et de condensat au niveau de Hassi Rmel

$P_{B}^{\mathrm{INAS}}$ et $P_{C}^{\mathrm{INAS}}$ production de brut et de condensat au niveau d'In Amenas

$V_{k}^{\text {raf }} \quad$ demande de la raffinerie $k$ en brut

$G_{i j}^{\text {nom }} \quad$ capacité de transit nominale de la ligne $R_{i j} \quad$ coefficient de fiabilité de la ligne $(i-j)$

La solution du problème ainsi posé, pour les niveaux de production du tableau 4 et sur la base des valeurs suivantes :

$$
\begin{gathered}
S_{C}-S_{B}=10 \$ / \mathrm{m}^{3} \\
S_{R}=30 \$ / \mathrm{m}^{3} \\
S_{E}=0,08 \mathrm{kWh}
\end{gathered}
$$

est donnée dans le tableau 8. Les chiffres entre parenthèses indiquent le nombre de réservoirs de $52000 \mathrm{~m}^{3}$ qu'il y a lieu d'installer.

Les variantes V3 et V4 tiennent compte des pénalités de transit par le territoire tunisien :

$$
C_{p e n}=S_{p}\left(X_{23}+Y_{23}\right)
$$

avec $S_{P}$, pénalités par tonne de produit transité.

Ces coûts supplémentaires sont introduits dans le fonctionnel (20), l'ensemble des contraintes restant inchangé.

Le modèle présenté peut également être :

- transformé en modèle multipériodique en vue d'établir un échéancier des réalisations ;

- utilisé pour la réorganisation optimale des flux en cas de perturbations endogènes (arrêt volontaire ou intempestif prolongé d'un équipement) ou exogènes (fluctuation de la demande).

\section{CONCLUSION}

Le réseau algérien de transport de brut et de condensat est un système complexe. Sa modélisation par la théorie des graphes et la mise à profit du problème du flot maximal offrent une alternative simple et efficace. La représentation du pompage par batch induit la notion de bi-flots. Cette difficulté est contournée par l'introduction d'arcs déconnectants, permettant de ramener le problème à un schéma classique à flot unique. Pour être efficace, en raison du vieillissement inégal des lignes, la procédure de modélisation du réseau doit inclure la fiabilité des éléments 
composants. La modélisation par le flot maximal, associée à des modèles locaux de fiabilité, permet :

- le diagnostic des capacités réelles du réseau ;

- la simulation de l'impact de toute décision de réaffectation des flux, de rénovation partielle ou de réhabilitation ;

- l'analyse de la flexibilité du réseau face aux perturbations endogènes et exogènes.

La solution dégagée par la résolution du problème du flot maximal est le plus souvent une distribution de flux compatibles. En conséquence, l'extension optimale du réseau, en raison de la non-linéarité des coûts, est définie par l'intermédiaire d'un modèle de programmation non-linéaire.

\section{RÉFÉREN CES}

1 Masseron, J. (1982) Économie des hydrocarbures, Technip.

2 Mesarovic, M.D., Macko, D. et Takahara, Y. (1970) Theory of Hierarchical Multilevel Systems, Academic Press, New York.

3 Moisseev, N.N. (1985) Problèmes mathématiques d'analyse des systèmes, Mir, Moscou.

4 Hare, V.C. Jr. (1972) L'analyse des systèmes, outil moderne de gestion, Dunod.

5 Le Moigne, J.L. (1990) La modélisation des systèmes complexes, Dunod.

6 Smati, A. (1992) Approche systémique dans l'optimisation des systèmes technologiques complexes. MOAD'92, Bejaia, Algérie.

7 Smati, A. (1994) A Systemic Approach in Optimization of the Algerian Crude Oil Network. AMSE, Lyon, France.

8 Diaz-Garcia, A., Hogg, G.L. et Phillips, D.T. (1985) Combined Simulation and Network Optimization of Production/Distribution System. Simulation, février.

9 Ford, L.R. et Fulkerson, D.R. (1962) Flows in Networks, Princeton.

10 Gondran, M. et Minoux, M. (1995) Graphes et algorithmes, Eyrolles.

11 Hu, T.C. (1963) Multicommodity Network Flows. Operations Research, 11, 344-360.
12 Kennington, J.L. et Helgason, R.V. (1980) Algorithms for Network Programming, John Wiley \& Sons.

13 Rothschild, B. et Whinston, A. (1966) On Two-Commodity Network Flow. Operations Research, 14, 377-387.

14 Sakarovitch, M. (1973) Two Commodity Network Flows and Linear Programming. Mathematical Programming, 4, 1, 1-20.

15 Goldfarb, D. et Grigoriadis, M.D. (1988) A Computational Comparison of the Dinic and Network Simplex Methods for Maximum Flow. Annals of Operations Research, 13, 83-123.

16 Grigoriadis, M.D. (1986) An Efficient Implementation of the Network Simplex Method. Math. Prog. Study, 26, 83-92.

17 Aissani, A. (1992) Modèles stochastiques de la théorie de la fiabilité, OPU, Alger.

18 Yacin, E.M. et Berezine, V.L. (1972) Nadiojnost trouboprovodov, Nedra, Moscou (en russe).

19 Aunicky, Z. (1970) The Longitudinal Mixing of Liquids Flowing Successively in Pipeline. Canad. Jour. Chem. Eng., 48.

20 Lourier, M.V., Maron, V.I. et Youfin, B.A. (1979) Optimizatsia pocledovatelnoy perekatchki, Nedra, Moscou (en russe).

21 Sjenitzer, F. (1958) How Much Do Products Mixing in a Pipeline? Pipeline Eng., 30, 13.

22 Smati, A. et Ainouche, A. (1998) Optimisation du pompage par batch : cas de l'oléoduc HEH-Bejaia. JST Sonatrach, Alger.

23 Taylor, G. (1954) The Dispersion of the Matter in Turbulent Flow through a Pipe. Proc. Roy. Soc., 219, 1155.

24 Vincent-Genod, J. (1970) Transport des hydrocarbures liquides et gazeux par canalisation, Technip.

25 Cabet, R. et Lizoret, Y. (1974) Économie du transport par conduite, Technip.

26 Bekey, G.A. et Masri, S.F. (1983) Random Search Techniques for Optimization of Nonlinear Systems with many Parameters. Math. Comp. Simulation.

27 Smati, A. (1998) Nettoyage, diagnostic et réhabilitation des pipelines. Petrogas Consult, Alger.

28 Tiratsoo, J.N.H. (1992) Pipeline Pigging Technology, Gulf Publishing Company. 